INRA Prod. Anim., 2005, 18 (3), 183-192

\title{
Le phosphore dans la nutrition des porcs
}

\author{
C. JONDREVILLE, J.-Y. DOURMAD
}

INRA, Agrocampus Rennes, UMR, Systèmes d'Elevage, Nutrition Animale et Humaine, F-35590 Saint-Gilles

Courriel : Catherine.Jondreville@rennes.inra.fr

La diminution des rejets d'éléments polluants par les élevages de porcs et la limitation de l'utilisation des ressources non renouvelables sont des composantes essentielles d'une production durable de viande de porc. Au cours des dernières décennies des voies de diminution des rejets de Phosphore (P) par les porcs ont été explorées en raison de l’impact négatif de cet élément sur la qualité des eaux dans les zones à forte densité d'élevage. La diminution continuelle des réserves mondiales de phosphates motive également la limitation de leur utilisation dans l'alimentation des animaux.

La réduction des apports alimentaires de $\mathrm{P}$ aux porcs est une voie préventive de choix pour en limiter les rejets. Réglementaire dans certains pays de l’Union Européenne, elle est fortement conseillée en France où elle s'appuie sur les références établies par le CORPEN en 2003 qui permettent de prévoir les rejets de $\mathrm{P}$ en fonction des quantités de P ingérées par les animaux et proposent des stratégies d'alimentation adéquates.

Réduire les apports alimentaires de $\mathrm{P}$ aux porcs suppose un meilleur ajustement des apports alimentaires aux besoins des animaux et la recherche de voies d'amélioration de sa disponibilité. Les porcelets sevrés excrètent environ $52 \%$ du phosphore qu'ils ingèrent, les porcs en engraissement environ $64 \%$ et les truies près de $80 \%$ (Poulsen et al 1999). Pour toutes ces catégories d'animaux, environ $50 \%$ de $\mathrm{P}$ ingéré est excrété dans les fèces. Le reste, qui représente l'excès de $\mathrm{P}$ apporté par rapport aux besoins des animaux, est rejeté via l'urine. Les truies, rejettent ainsi par cette voie environ $27 \%$ du phosphore ingéré. Pour mettre en œuvre des stratégies de diminution des rejets de $\mathrm{P}$ par la voie alimentaire, il est donc nécessaire d'améliorer la digestibilité de $\mathrm{P}$ alimentaire pour toutes les catégories d'animaux et de mieux définir les besoins, notamment pour les reproducteurs.
En France, les bases d'estimation de la valeur $\mathrm{P}$ des aliments et des besoins des animaux s'appuient sur le concept de digestibilité réelle de $P$. Les bases scientifiques sur lesquelles repose ce système ont été détaillées par Guéguen et Pointillart (1986). Au cours des dernières décennies, de nombreux travaux ont porté sur la mesure de la digestibilité apparente de $\mathrm{P}$ des matières premières. Le présent article fait le point sur les résultats récents, tout en gardant les acquis antérieurs, en vue d'élaborer un système cohérent d'évaluation de la valeur $\mathrm{P}$ des aliments et de détermination des besoins des animaux selon leur stade physiologique. Par ailleurs, différentes voies d'amélioration de la digestibilité de $\mathrm{P}$ chez le porc sont présentées, notamment l'usage de phytase microbienne.

\section{1 / Valeur $\mathbf{P}$ des matières premières et des aliments chez le porc}

\section{1 / Bases d'estimation des apports nutritionnels de $\mathbf{P}$ par les matières premières et les ali- ments}

La première étape pour caractériser la valeur $\mathrm{P}$ d'une matière première ou d'un aliment est d'en déterminer la teneur en $\mathrm{P}$ total, mais cette grandeur ne rend compte ni de la nature chimique du phosphore ni de sa disponibilité pour l'animal. Aussi, au cours des vingt dernières années, de nombreuses études ont porté sur l'évaluation de la valeur $\mathrm{P}$ des matières premières et des aliments au-delà de leur simple teneur en P. Différents concepts sont utilisés.

a) Les concepts utilisés : digestibilité apparente, disponibilité

Les tables de valeurs nutritionnelles des matières premières ont été construites à partir de deux modes d'évaluation de la valeur $\mathrm{P}$ : d'une part, la digestibilité apparente, mesurée à la fin du tube digestif, parfois appelée digestibilité fécale apparente ou digestibilité totale apparente, et d'autre part la disponibilité. La première prévaut dans les tables néerlandaises (CVB 2000) et françaises (INRA-AFZ 2004) alors que la seconde est privilégiée dans les tables nord américaines (NRC 1998). Dans le premier cas, on ne s'intéresse qu'au compartiment digestif. Dans le second cas, il s'agit d'une utilisation globale de la source de P étudiée, l'indicateur pouvant être la minéralisation osseuse. Les deux types de valeurs, même s'ils aboutissent à la même hiérarchie de matières premières, ne peuvent pas être utilisés simultanément pour formuler un aliment.

En Europe occidentale, le concept de digestibilité apparente prévaut et c'est celui que nous allons privilégier dans cet article. Les nombreuses mesures effectuées au cours des vingt dernières années ont montré la grande variabilité de la digestibilité de $\mathrm{P}$ entre matières premières (19 \% pour le tourteau de tournesol à $90 \%$ pour des coproduits laitiers, en passant par $47 \%$ pour le pois, selon les tables INRA-AFZ 2004). La prise en compte de ces données est donc impérative pour réduire les marges de sécurité lors de la formulation des aliments pour porcs.

Bien que le concept de $\mathrm{P}$ digestible apparent constitue un progrès très important par rapport à l'utilisation de la teneur en P total, certains auteurs en soulignent les limites. La digestibilité apparente du phosphore contenu dans un même lot de tourteau de soja varie ainsi de 38 à $45 \%$ quand son pourcentage d'incorporation dans un aliment de base ne contenant pas de $\mathrm{P}$ varie de 41 à 55 \% (Fan et al 2001). De même, la digestibilité apparente de $\mathrm{P}$ d'un lot de maïs varie de 22 à $39 \%$ lorsque son pourcentage d'incorporation dans un aliment de base sans $\mathrm{P}$ augmente de 54 à 72 \% (Shen et al 2002). La proportion variable des pertes endogènes basales de $\mathrm{P}$ dans l'excrétion totale de $\mathrm{P}$, qui explique ces différentes valeurs 
de digestibilité, contribuerait à la variabilité de la digestibilité de $\mathrm{P}$ intra matière première et expliquerait en partie la non additivité des valeurs de $\mathrm{P}$ digestible apparent (Fan et Sauer 2002). Afin de pallier ce biais méthodologique, la digestibilité apparente devrait donc être corrigée par des pertes endogènes basales de $P$, selon le principe utilisé pour la correction des valeurs de digestibilité apparente des acides aminés (Sève et al 2000). Comme le soulignent ces auteurs, cette correction n'a pas pour objectif de distinguer le $\mathrm{P}$ d'origine endogène du $\mathrm{P}$ d'origine alimentaire dans l'excrété total, ce qui aboutirait au calcul d'une digestibilité réelle. Elle a pour but de rendre indépendante la valeur $\mathrm{P}$ attribuée à la matière première de son taux d'incorporation dans l'aliment expérimental. Cette piste mérite d'être explorée. Cependant, une telle correction requiert l'établissement préalable d'une valeur de pertes endogènes basales de P. Par ailleurs, elle n'est efficace que lorsque la digestibilité est mesurée en diluant la matière première objet de l'étude dans un aliment de base ne contenant pas de P. Lorsque la digestibilité est calculée par différence, en mélangeant la matière première objet de l'étude avec, le plus souvent, un lot de maïs dont la digestibilité de $\mathrm{P}$ est connue, ce biais méthodologique n'existe pas. Enfin, l'impact de la correction envisagée sur la variabilité intra matière première et l'additivité des valeurs de $\mathrm{P}$ digestible reste à évaluer.

b) Facteurs de variation de la digestibilité de $P$ des matières premières et des aliments

- Nature du phosphore présent dans la ration

Le phosphore présent dans les aliments pour les porcs provient des matières premières et des phosphates ajoutés dans la ration. Toutes les tables soulignent la variabilité de la digestibilité de $\mathrm{P}$ entre phosphates. Dans un contexte de limitation des rejets de $\mathrm{P}$ par les porcs, l'utilisation des phosphates les plus digestibles devrait donc être favorisée. Par exemple, indépendamment de toute considération économique, le phosphate monocalcique devrait être préféré au phosphate bicalcique. Les tables font également état de la variabilité de la digestibilité de $P$ intra phosphate et encouragent à une bonne connaissance du produit utilisé, afin d'éviter des apports insuffisants de $\mathrm{P}$ ou des rejets excessifs de cet élément.
Le phosphore présent dans les matières premières d'origine végétale est sous forme phytique ou non phytique (phosphoprotéines, phospholipides, nucléoprotéines...). Le phosphore phytique est la forme majeure de stockage de $\mathrm{P}$ dans les matières premières d'origine végétale et en représente 50 à 80 \% (Pointillart 1994). Le phosphore d'origine phytique présente une faible digestibilité chez les animaux monogastriques qui ne disposent pas de l'équipement enzymatique nécessaire à la déphosphorylation des phytates. La digestibilité de $\mathrm{P}$ d'une matière première d'origine végétale dépend donc en premier lieu des proportions de P phytique et non phytique dans $\mathrm{P}$ total. Des augmentations substantielles de la digestibilité de $\mathrm{P}$ ont ainsi été obtenues en développant des variétés de maïs et d’orge dites «low phytate» présentant une proportion de $\mathrm{P}$ phytique dans $\mathrm{P}$ total de l'ordre de 35-40 \% (Spencer et al 2000, Veum et al 2001 et 2002, Skiba et al 2004) au lieu de 60-75\% pour les variétés habituelles. A partir d’une base de données composée de quarante lots de matières premières sur lesquels les teneurs en $\mathrm{P}$ total, $\mathrm{P}$ phytique, activité phytique et digestibilité de P ont été mesurées selon une méthodologie standardisée (Skiba et al 2004), la digestibilité de $\mathrm{P}$ phytique et celle de $\mathrm{P}$ non phytique ont été estimées à respectivement 0,23 et 0,45 . Guéguen et Perez (1981) estiment la digestibilité réelle de $\mathrm{P}$ phytique à 0,35 mais nos estimations diffèrent des 0,10 et 0,90 pour la digestibilité respectivement de $\mathrm{P}$ phytique et de $\mathrm{P}$ non phytique estimés par Weremko et al (1997) à partir de données de la littérature. De telles différences méritent qu'on s'attarde davantage sur l'estimation de ces coefficients de digestibilité et sur leurs facteurs de variation. La solubilité des phytates et leur localisation dans le grain pourraient en faire partie. Par exemple, dans le blé, plus de $80 \%$ des phytates sont localisés dans les enveloppes externes, $90 \%$ sont concentrés dans le germe de maïs alors qu'ils sont localisés dans toutes les parties de la graine de soja (Pointillart 1994). Les estimations ci-dessus devraient alors être établies par famille de matières premières.

- Phytases végétales, phytases microbiennes

Les phytases sont des enzymes qui permettent l'hydrolyse de la molécule de phytate et la libération des groupes phosphate qui y sont liés. Elles appartiennent à deux groupes distincts : les 3-phytases et les 6-phytases qui com- mencent la déphosphorylation en positions respectivement 3 et 6 . En règle générale, la faible activité phytasique présente dans la partie proximale du tube digestif des porcs, provenant de l'intestin ou de sa flore, est sans effet significatif sur la digestibilité de $\mathrm{P}$ phytique. Récemment, un porc exprimant une phytase salivaire a montré sa capacité à libérer pratiquement en totalité, et dans la partie proximale de l'intestin grêle, le phosphore phytique (Golovan et al 2001). Mais l'utilisation de phytases, végétales ou microbiennes, demeure la voie de choix pour améliorer la digestibilité de P. Les phytases végétales appartiennent au groupe des 6-phytases alors qu'il existe des 3-phytases et des 6-phytases d'origine microbienne. Ces enzymes permettent de libérer les groupes phosphates des phytates dans la partie proximale de l'intestin, avant les sites d'absorption, et donc d'améliorer la digestibilité de P. Une activité phytasique substantielle d'origine microbienne est également mesurée dans le côlon des porcs. Mais elle ne permet la déphosphorylation des phytates qu'après les sites d'absorption et, par conséquent, est inefficace en terme d'amélioration de la disponibilité de $\mathrm{P}$ chez le porc (Schlemmer et al 2001).

Les phytases, d’origine végétale ou microbienne, sont caractérisées par une activité phytasique mesurée in vitro. Une unité d'activité phytasique est définie comme la quantité de phytase qui permet de libérer $1 \mu \mathrm{mol}$ de $\mathrm{P}$ inorganique d'une solution contenant $5,1 \mathrm{mmol}$ de phytate de sodium / l, à $\mathrm{pH} 5,5$ et $37^{\circ} \mathrm{C}$ (Engelen et al 1994). Le pH optimum des phytases, microbienne (3-phytase) et végétale, est aux alentours de 5-5,5. L'activité de la phytase végétale diminue très rapidement lorsque le $\mathrm{pH}$ est abaissé, alors que l'activité de la phytase microbienne (3-phytase) est encore aux alentours de $60 \%$ à pH 2 (Eeckhout et De Paepe 1992). Rapp et al (2001) ont montré que la phytase microbienne (3-phytase) résiste mieux à la dénaturation dans l'estomac que la phytase végétale, puisque $70 \%$ de son activité est préservée dans l'intestin grêle de mini-porcs, contre 40-45 \% pour de la phytase de blé. Ces facteurs expliquent au moins en partie pourquoi, pour une même activité mesurée in vitro, la phytase végétale est 1,4 à 2,5 fois moins efficace in vivo que la phytase microbienne (3-phytase) (Eeckhout et De Paepe 1992, Weremko et al 1997, Zimmermann et al 2002). 


\section{Phytases végétales}

La phytase végétale est présente en quantités variables dans les graines de céréales à paille et leurs coproduits (seigle, triticale, blé, orge, son de blé...). Au contraire, le maïs, l'avoine et les tourteaux d'oléagineux (tourteau de soja, tourteau de colza...) en sont dépourvus (Eeckhout et De Paepe 1994, Pointillart 1994). L'activité phytasique est donc variable non seulement entre matières premières mais également intra matière première. Par exemple, Eeckhout et De Paepe (1994) ont mesuré une activité allant de 915 à $1581 \mathrm{U} / \mathrm{kg}$ et de 1180 à $5208 \mathrm{U} / \mathrm{kg}$ dans respectivement 13 lots de blé et 5 lots de son de blé. De plus, des éléments tels que la durée de stockage, la présence de cations dans la ration, comme le calcium qui stabilise les phytates, ou la taille des particules sont autant de facteurs de variation de l'efficacité de la phytase végétale pour une même activité mesurée in vitro (Nys et al 1996). Le trempage d'aliments à base de blé ou d'orge permet d'améliorer l'efficacité des phytases végétales et de diminuer leur teneur en phytates avant ingestion par les animaux (Skoglund et al 1997, Larsen et al 1999, Carlson et Poulsen 2003) mais est parfois sans effet sur la digestibilité de P mesurée in vivo (Näsi et al 1995, Skoglund et al 1997, Larsen et al 1999). Selon Larsen et al (1999) cette absence d'effet sur la digestibilité serait due à la diminution de l'activité phytasique en raison d'une protéolyse accrue par le trempage.

Quoi qu'il en soit, l'introduction de matières premières présentant une activité phytasique intrinsèque élevée est une voie d'amélioration de la disponibilité de P phytique non seulement de la matière première en question mais des autres ingrédients constitutifs de la ration (Pointillart 1991). Cependant, elle n'est efficace qu'en l'absence de traitement thermique de l'aliment puisqu'elle est presque totalement inactivée à des températures atteignant $70^{\circ} \mathrm{C}$.
D’autres voies d'amélioration de la digestibilité de P doivent être adoptées en cas de traitement thermique.

\section{Phytases microbiennes}

Pour les aliments granulés, la principale stratégie est l'utilisation de phytases microbiennes. Actuellement, quatre phytases sont autorisées dans l'alimentation des porcs de l'Union Européenne, trois 3-phytases et une 6-phytase. La réponse de $\mathrm{P}$ digestible à des niveaux croissants de phytase est curvilinéaire et la dégradation de $\mathrm{P}$ phytique avant les sites d'absorption n'excède jamais 60-70 \%, même à des niveaux élevés de phytase ajoutée. Des équations d'équivalence entre phytase microbienne et $\mathrm{P}$ digestible apparent ont été établies à partir des données de la littérature (tableau 1). Tous les auteurs de ces méta-analyses soulignent la variabilité de la réponse pour une même activité phytasique. L'utilisateur de ces équations doit être conscient que la plupart des études ont porté sur une 3-phytase introduite dans des aliments pour porcelets ou porcs en engraissement composés de maïs et de tourteau de soja, donc dépourvus de phytase d'origine végétale. Dans ces conditions, ces équations suggèrent que 500 unités de phytase microbienne (3-phytase) sont équivalentes à 0,65-0,75 $\mathrm{g}$ de $\mathrm{P}$ digestible apparent.

Lorsque l'aliment est à base de blé ou d'orge, l'équivalence est moindre et est réduite à $0,43 \mathrm{~g}$ de $\mathrm{P}$ digestible pour 500 unités de phytase microbienne (Johansen et Poulsen 2003). Les caractéristiques des phytates présents dans la ration pourraient être à l'origine de cette différente efficacité des phytases (Nys et al 1996). Mais très probablement, il est dû à l'action des phytases végétales, dont les effets sont additifs avec ceux de la phytase microbienne (Zimmermann et al 2003). Ces résultats suggèrent d'adapter les équivalences au type d'aliment. En particulier, l'équivalence entre phytase microbienne et
$\mathrm{P}$ digestible doit être réduite lorsque l'aliment contient des matières premières présentant une activité phytasique intrinsèque significative.

D’autres éléments doivent être pris en compte. Pour une même activité mesurée in vitro, différents produits commerciaux sont susceptibles de libérer des quantités différentes de P digestible, indépendamment de la classe (3 ou 6-phytase) (Augspurger et al 2003). Chaque phytase microbienne est donc normalement assortie d'une équation d'équivalence spécifique. La présence de cations divalents en quantités importantes dans la ration, tels que le calcium ou le zinc apporté à doses pharmacologiques qui stabilisent les phytates, limite l'efficacité des phytases. Chez le porcelet sevré recevant un aliment contenant 3,6 g de P et supplémenté avec 700 U de phytase microbienne, Qian et al (1996) ont observé une diminution de la digestibilité de $\mathrm{P}$ de 0,53 à 0,44 lorsque le rapport $\mathrm{Ca} / \mathrm{P}$ augmente de 1,2 à 2,0. A cet égard, la diminution de la teneur en Ca des aliments contenant de la phytase microbienne est recommandée. Cependant, si l'effet positif de la phytase sur la disponibilité de Ca est avéré, peu de données expérimentales permettent d'établir une équivalence entre $\mathrm{Ca}$ et phytase microbienne. Selon Kornegay (2001), 500 unités de 3-phytase correspondent à 0,4 à $1,1 \mathrm{~g}$ de Ca. Plus récemment, l'effet négatif de l'utilisation de zinc à dose pharmacologique dans l'alimentation du porcelet sur l'efficacité des phytases microbiennes a été démontré (Augspurger et al 2004). Certains traitements comme le trempage améliorent l'efficacité des phytases microbiennes (Näsi et al 1995, Liu et al 1997). Des effets synergiques entre l'acide citrique (Kemme et al 1999), l'acide formique (Jongbloed et al 2000) et les phytases microbiennes ont été observés. Mais ces effets n'ont pas été montrés avec l'acide citrique (Radcliffe et al 1998) et un mélange d'acides (Omogbenigun et al 2003).

Tableau 1. Equations d'équivalence entre $P$ digestible $(g)$ et phytase microbienne (Phyt., Unités).

\begin{tabular}{|c|c|c|c|c|}
\hline & Phytase ajoutée (Unités) & 250 & 500 & 750 \\
\hline Référence & Equation d'équivalence & \multicolumn{3}{|c|}{ Equivalence } \\
\hline Düngelhoef et Rodehutscord (1995) & $0,99\left(1-\mathrm{e}^{-0,0025 \times \text { Phyt }}\right)$ & 0,46 & 0,71 & 0,84 \\
\hline Kornegay (2001) & $1,026\left(1-e^{-0,00263 \times \text { Phyt }}\right)$ & 0,49 & 0,75 & 0,88 \\
\hline Jean dit Bailleul et al (2001) & $1,67 \times 10^{-3} \times$ Phyt $6,49 \times 10^{-7} \times$ Phyt $^{2}$ & 0,38 & 0,67 & 0,89 \\
\hline Johansen et Poulsen (2003) ${ }^{(1)}$ & $0,86\left(1-e^{-0,0028 \times \text { Phyt }}\right)$ & 0,43 & 0,65 & 0,75 \\
\hline Johansen et Poulsen (2003) ${ }^{(2)}$ & $0,82\left(1-e^{-0,0015 \times \text { Phyt }}\right)$ & 0,26 & 0,43 & 0,55 \\
\hline
\end{tabular}

(1) Aliments à base de maïs.

(2) Aliments à base de céréales à paille. 


\section{- Facteurs liés à l’animal}

La plupart du temps, la digestibilité apparente est mesurée sur des porcs en croissance alimentés en dessous de leur besoin en P. Dans ces conditions, les pertes endogènes de $\mathrm{P}$ sont minimisées et l'absorption est maximisée. Ainsi, la valeur de digestibilité attribuée à une matière première est une valeur potentielle.

La question de la pertinence des valeurs de digestibilité apparente obtenues sur des porcs de 40-45 kg pour la formulation d'aliments pour les porcelets sevrés et les truies se pose. L'effet du poids de l'animal, de son âge ou de son stade physiologique sur la digestibilité de $\mathrm{P}$ d'une même matière première ou d'un aliment pourrait être dû à une variation de la proportion des pertes endogènes dans l'excrété fécal de $\mathrm{P}$ et/ou à une adaptation de l'absorption de l'animal en regard de la couverture de ses besoins. La connaissance et la quantification de ces phénomènes permettraient de corriger les valeurs de digestibilité tabulées ou d'adapter les termes du calcul factoriel du besoin aux différents stades physiologiques.

La digestibilité plus élevée de $\mathrm{P}$ des matières distribuées aux jeunes animaux et l'augmentation de l'apport par rapport au besoin chez l'animal plus lourd ou plus âgé ont parfois été interprétées comme un effet négatif de l'âge ou du poids sur la digestibilité apparente de P. En réalité, chez des porcs en engraissement alimentés en dessous de leur besoin en $\mathrm{P}$, la digestibilité de $\mathrm{P}$ augmente avec le poids des animaux et se stabilise aux environs de $60 \mathrm{~kg}$ (Eeckhout et al 1995, Kemme et al 1997a et b). Selon Rodehutscord et al (1999), la digestibilité de $\mathrm{P}$ de matières premières d'origine végétale mesurée chez des porcs de $40 \mathrm{~kg}$ peut être utilisée pour formuler des aliments pour porcelets sevrés. La question de l'adaptation des valeurs tabulées à d'autres stades physiologiques se pose davantage pour les truies, notamment en gestation. Kemme et al (1997b) ont montré que la digestibilité de $\mathrm{P}$ d'un même aliment est la plus élevée chez les porcs en croissance, suivis des truies en lactation puis des truies en milieu de gestation. Cette faible digestibilité chez la truie en milieu de gestation est en partie expliquée par le faible besoin en $\mathrm{P}$ de cet animal, mais d'autres données expérimentales doivent être acquises avant la mise en place d'un système de correction adéquat.
Comme pour les valeurs de digestibilité, se pose la question de l'adaptation à différents stades physiologiques des équivalences entre phytase microbienne et $\mathrm{P}$ digestible apparent. Rodehutscord et al (1999) ont mesuré la même efficacité de 400 unités d'une 3-phytase chez des porcs pesant 16 et $40 \mathrm{~kg}$. Mais selon Kemme et al (1997a) l'efficacité d'une 3-phytase est plus élevée chez la truie en lactation et le porc en engraissement que chez le porcelet sevré (respectivement 1 ; 0,8 et 0,7 g $\mathrm{P}$ digestible apparent pour 500 unités). L'équivalence est de loin la plus faible chez la truie en milieu de gestation avec $0,32 \mathrm{~g}$ de $\mathrm{P}$ digestible apparent pour 500 unités. Jongbloed et al (2004) ont publié des résultats similaires à propos d'une 6-phytase : 750 unités étaient équivalentes à 0,8 ; 0,3 et 0,4 g $\mathrm{P}$ digestible apparent chez respectivement la truie en lactation, la truie à 70 jours de gestation et la truie à 100 jours de gestation. Ces auteurs suggèrent d'adapter les équivalences au stade physiologique, notamment pour les truies en gestation, mais les données expérimentales sont encore trop peu nombreuses.

Un nombre important de travaux a été conduit afin d'établir la valeur $\mathrm{P}$ des matières premières et de quantifier l'effet des phytases sur l'amélioration de la digestibilité de $\mathrm{P}$ phytique. Bien qu'encore incomplets, ces travaux permettent d'ores et déjà de concevoir un système additif de formulation sur la base du phosphore digestible apparent.

\section{2 / Estimer la teneur en $\mathbf{P}$ digestible apparent d'un aliment pour porcs}

a) Valeur $P$ des phosphates et des matières premières

Les tables INRA-AFZ 2004 présentent des Valeurs Biologiques Relatives

Tableau 2. Valeur Biologique Relative (VBR) et digestibilité apparente (dP) des principaux phosphates utilisés dans l'alimentation des porcs.

\begin{tabular}{|l|c|c|}
\hline \multicolumn{1}{|c|}{ Phosphates } & VBR $(\%)^{(1)}$ & dP $(\%)^{(2)}$ \\
\hline Monosodique & 100 & 90 \\
Bicalcique anhydre & 73 & 66 \\
Bicalcique hydraté & 77 & 69 \\
Monocalcique & 92 & 83 \\
Monobicalcique & 83 & 75 \\
Monosodique feed grade & 98 & 88 \\
Triple Ca, Mg, Na & 93 & 84 \\
\hline
\end{tabular}

(1) Valeurs moyennes (Tables INRA-AFZ 2004). Dans ces tables, sont indiqués le nombre d'échantillons utilisés pour générer ces valeurs moyennes et leur variabilité.

(2) $\mathrm{dP}=\mathrm{VBR} \times 0,90$.
(VBR) avec le phosphate monosodique comme référence. Le calcul de la digestibilité apparente des phosphates à partir de leur VBR consiste à attribuer à la source de référence non pas la valeur $100 \%$, mais sa digestibilité apparente, qui est de $90 \%$. Le tableau 2 présente les valeurs de digestibilité apparente de $\mathrm{P}$ des principaux phosphates utilisés dans l'alimentation des porcs.

Afin de rendre compte de la dénaturation de la phytase végétale dans les aliments ayant subi un traitement thermique, les tables INRA-AFZ 2004 proposent deux valeurs de digestibilité apparente de $\mathrm{P}$ : l'une (dP) correspondant à une matière première dont la phytase intrinsèque est dénaturée et l'autre (dPphy) correspondant à la matière première native. Pour plus de commodité, ces deux valeurs de digestibilité sont dénommées respectivement «granulé» et «farine». Pour les matières premières à activité phytasique significative, comme les céréales à paille et leurs coproduits, dPphy est supérieure à dP. Par exemple, la digestibilité de $\mathrm{P}$ du blé tendre est de $30 \%$ dans un aliment granulé et de $45 \%$ dans un aliment de type farine. Lorsque la matière première présente une activité phytasique négligeable, comme le maïs par exemple, ces deux valeurs sont égales. En d'autres termes, la digestibilité de $\mathrm{P}$ est la même, que cette matière première soit introduite dans un aliment granulé ou distribué sous forme de farine (tableau 3).

b) Prendre en compte la présence de phytase dans les aliments

\section{Phytase végétale}

La prise en compte de la phytase végétale ne concerne que les aliments n'ayant pas subi de traitement thermique. Compte tenu de la variabilité de l'activité phytasique des céréales à paille (Eeckhout et De Paepe 1994, Pointillart 1994), il est raisonnable de 
Tableau 3. Valeur $P$ de quelques matières premières entrant dans la composition des aliments pour porcs $(\mathrm{g} / \mathrm{kg}$ de matière première) $)^{(1)}$.

\begin{tabular}{|l|c|c|c|c|c|}
\hline & \multirow{2}{*}{$\mathbf{P}$ total $\mathbf{g} / \mathbf{k g})$} & \multicolumn{2}{|c|}{ Granulé } & \multicolumn{2}{|c|}{ Farine } \\
\cline { 3 - 6 } & & $\mathrm{dP}(\%)^{(2)}$ & $\mathrm{P}$ dig. $(\mathrm{g} / \mathrm{kg})^{(2)}$ & $\mathrm{dPPhy}(\%)^{(3)}$ & $\mathrm{P}$ digPhy $(\mathbf{g} / \mathrm{kg})^{(3)}$ \\
\hline Blé & 3,2 & 30 & 0,96 & 45 & 1,4 \\
Triticale & 3,5 & 30 & 1,05 & 48 & 1,7 \\
Son de blé & 9,9 & 25 & 2,5 & 50 & 5,0 \\
Maïs & 2,6 & 28 & 0,73 & 28 & 0,73 \\
Tourteau de soja 48 & 6,2 & 32 & 2,0 & 32 & 2,0 \\
Phosphate monocalcique & 230 & 83 & 191 & 83 & 191 \\
Phosphate bicalcique hydraté & 175 & 69 & 121 & 69 & 121 \\
Phytase Niveau 1 (0-250 U) $(4)$ & 0 & - & 10000 & - & 10000 \\
Phytase Niveau 2 (250-500 U) & 0 & - & 5000 & - & 5000 \\
Phytase Niveau 3 (500-750 U) & 0 & - & 2600 & - & 2600 \\
Phytase Niveau 4 (750-1000 U) & 0 & - & 1400 & - & 1400 \\
\hline
\end{tabular}

(1) Tables INRA-AFZ 2004.

(2) dP, digestibilité fécale apparente phytase dénaturée par un traitement de granulation par exemple ; P dig., teneur en P digestible apparent

$=\mathrm{P}$ total $\mathrm{x} \mathrm{dP}$.

(3) dPPhy, digestibilité fécale apparente phytase non dénaturée (aliment distribué en farine) ; P digPhy., teneur en P digestible apparent

$=\mathrm{P}$ total $\mathrm{x}$ dPPhy.

(4) Phytase : phytase microbienne, produit formulé pour contenir 5000 Unités / g. Le pourcentage d'incorporation de chacun des produits est plafonné à $0,05 \mathrm{~g}$ soit 250 unités / kg d'aliment. Les équivalences, obtenues à partir de l'équation établie par Kornegay (2001) présentée au tableau 1, sont de respectivement 0,$196 ; 0,104 ; 0,052$ et 0,028 g P digestible apparent pour 100 unités de phytase Niveaux $1,2,3$ et 4 .

ne comptabiliser la quantité de P libéré en termes de $\mathrm{P}$ digestible apparent qu'en deçà de 500 unités de phytase végétale par kg d'aliment, soit au maximum 0,4 g P digestible apparent par kg d'aliment. Ainsi, pour obtenir un aliment contenant 2,5 g de $\mathrm{P}$ digestible apparent / kg, il faut s'assurer que sa teneur en $\mathrm{P}$ digestible apparent calculée à partir de dPPhy (farine) est d'au moins 2,5 g / kg et que sa teneur en P digestible apparent calculée à partir de dP (granulé) est d'au moins 2,1 g / kg (tableau 4).

\section{Phytase microbienne}

La valorisation des phytases microbiennes repose sur des équations d'équivalences qui doivent être établies pour chaque produit. Dans l'état actuel des connaissances, nous ne prenons pas en compte le possible effet du stade physiologique sur ces équivalences.

Les équations présentées au tableau 1 permettent de traduire l'apport de phytase microbienne dans un aliment sans phytase naturelle en termes de $P$ digestible apparent. Par exemple, à partir de l’équation établie par Kornegay (2001) on peut estimer que jusqu'à 500 unités,
100 unités de phytase microbienne correspondent à $0,15 \mathrm{~g}$ de $\mathrm{P}$ digestible apparent, soit une équivalence de $0,75 \mathrm{~g}$ de $\mathrm{P}$ digestible apparent pour 500 unités de phytase microbienne. Audelà, l'efficacité de la phytase microbienne par unité est moindre, et l'équivalence est de $0,040 \mathrm{~g}$ de $\mathrm{P}$ digestible apparent par 100 unités de phytase supplémentaire. Le nombre d'équivalences peut être augmenté afin de mieux représenter la curvilinéarité de la réponse. En formulation, cela revient à introduire autant de phytases microbiennes que d’équivalences, même si le même produit est utilisé. Un exemple avec quatre valeurs (Niveau 1 : 0-250 U, Niveau 2 : 250-500 U, Niveau 3 : 500-750 U et Niveau 4 : 750-1000 U) est présenté au tableau 3.

En présence de phytases naturelles non dénaturées (aliment type farine), ces équivalences doivent être adaptées, compte tenu de l'additivité des activités phytasiques végétale et microbienne (Johansen et Poulsen 2003, Zimmermann et al 2003). Il faudra alors plafonner la quantité de $\mathrm{P}$ libéré par la phytase endogène et la phytase microbienne Niveau 1 à 0,5 g de

Tableau 4. Valorisation de la phytase végétale - contraintes minimales pour un aliment contenant 2,5 g de $P$ digestible apparent par kilogramme.

\begin{tabular}{|l|c|c|}
\hline \multicolumn{1}{|c|}{ Présentation } & Granulé & Farine \\
\hline P digestible apparent, dP (g / kg aliment) & 2,5 & 2,1 \\
P digestible apparent, dPphy (g / kg aliment) & 2,5 & 2,5 \\
\hline
\end{tabular}

$\mathrm{P}$ digestible apparent / kg dans l'exemple du tableau 3. Ainsi, l'efficacité de la phytase microbienne sera plus rapidement réduite avec le niveau d'incorporation en présence qu'en l'absence de phytase endogène.

\section{2 / Estimation du besoin en $P$ digestible apparent de l'animal en croissance, de la truie en gestation et de la truie en lactation}

Le besoin en $\mathrm{P}$ du porc est souvent estimé selon une approche factorielle qui consiste à additionner les besoins estimés pour l'entretien, la croissance et la production (ARC 1981, Guéguen et Perez 1981, Guéguen et Pointillart 1986, Jongbloed et al 1999).

\section{1 / Les bases du calcul factoriel du besoin en $P$ digestible appa- rent}

Les équations de base utilisées pour le calcul factoriel du besoin en P digestible apparent sont présentées au tableau 5.

\section{a) Besoin d'entretien}

La méthode décrite par Guéguen et Perez (1981) et par Sauveur et Perez dans les tables INRA en 1989 repose 
Tableau 5. Bases de la détermination factorielle du besoin en $P$ digestible apparent.

\begin{tabular}{|c|c|c|}
\hline \multicolumn{3}{|r|}{ Porcelet sevré, porc en engraissement } \\
\hline Entretien & $P$ endogène (mg / jour) & $10 \times \mathrm{PV}$ \\
\hline Croissance & P corporel $(g)^{(1)}$ & $-0,002857 \times \mathrm{PV}^{2}+5,4199 \times \mathrm{PV}$ \\
\hline \multicolumn{3}{|r|}{ Truie en lactation } \\
\hline Entretien & P endogène (mg / jour) & $10 \times P V$ \\
\hline Lactation & $P$ sécrété via le lait $(g / \text { jour })^{(2)}$ & $(0,0257 \times$ Croissance Portée $+0,42 \times$ NPV $) \times 6,38 \times 1,55 / 50$ \\
\hline \multicolumn{3}{|r|}{ Truie en gestation } \\
\hline Entretien & $P$ endogène (mg / jour) & $10 \times P V$ \\
\hline Croissance & P corporel $(g)^{(1)}$ & $-0,002857 \times P V^{2}+5,4199 \times P V$ \\
\hline Gestation & $\begin{array}{l}\text { P dans les foetus }(\mathrm{g})^{(3)} \\
\text { P dans le placenta }(\mathrm{g})^{(4)}\end{array}$ & $\begin{array}{l}\operatorname{Exp}[4,591-6,389 \times \operatorname{Exp}(-0,023998 \times(j-45))+0,0897 \times N P T] \times \text { PdsPortée } \times 6,25 / \\
\operatorname{Exp}[4,591-6,389 \times \operatorname{Exp}(-0,023998 \times(115-45))+0,0897 \times N P T] \\
\operatorname{Exp}[7,34264-1,40598 \times \operatorname{Exp}(-0,06250 \times(j-45))+0,00759 \times j \\
+0,06339 \text { NPT }] \times 0,0096 / 23,8\end{array}$ \\
\hline
\end{tabular}

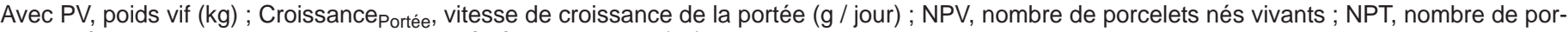
celets nés totaux; Pds portée, poids de la portée à la naissance $(\mathrm{kg})$; j, jour de gestation.

(1) Calculé à partir de données de la littérature.

(2) Calculé à partir de la quantité d'azote sécrété dans le lait estimée par Noblet et Etienne (1989), avec 6,38 g de protéine par g d'azote, 50 g de protéine et $1,55 \mathrm{~g}$ de $\mathrm{P} / \mathrm{kg}$ de lait (Guéguen et Perez 1981).

(3) Jongbloed et al (1999).

(4) D'après l'estimation de l'accrétion protéique (kJ) par Noblet et al (1985), avec 0,96 g de P pour 100 g de protéine et 23,8 kJ / g de protéine.

sur la digestibilité réelle de $\mathrm{P}$ : le besoin en $\mathrm{P}$ digestible réel est calculé puis exprimé sur la base du phosphore total en estimant que la digestibilité réelle potentielle de $\mathrm{P}$ est en moyenne de $50 \%$ pour un régime classique à base de maïs et de tourteau de soja complété en phosphate calcique. Selon ce système, le besoin d'entretien correspond à la somme des pertes endogènes fécales et urinaires obligatoires de P. Or, lorsque l’aliment est caractérisé par sa teneur en $\mathrm{P}$ digestible apparent, les pertes endogènes fécales de $\mathrm{P}$ sont déjà comptabilisées dans la valeur P qui lui est attribuée. Le besoin d'entretien en $\mathrm{P}$ digestible apparent est donc égal aux seules pertes endogènes urinaires obligatoires de $\mathrm{P}$.

Ce calcul constitue toutefois une approximation. En effet, les pertes endogènes fécales de $\mathrm{P}$ quotidiennes diminuent de $9 \mathrm{mg} / \mathrm{kg}$ de Poids Vif (PV) chez des animaux recevant un apport adéquat de $\mathrm{P}$ à $3 \mathrm{mg} / \mathrm{kg}$ PV chez des animaux en subcarence (Jongbloed et al 1999). Or, la digestibilité apparente de $\mathrm{P}$ des matières premières est souvent mesurée chez des animaux en subcarence. Leurs pertes endogènes fécales sont donc minimisées par rapport à des animaux en élevage, dont le besoin en $\mathrm{P}$ est normalement couvert. C'est pourquoi le besoin quotidien d'entretien est estimé au niveau assez élevé de $10 \mathrm{mg} /$ kg PV (Guéguen et Perez 1981), quel que soit le stade physiologique de l'animal. Jongbloed et al (1999) esti- ment ce besoin à un niveau légèrement inférieur de 7 mg P / kg PV.

\section{b) Besoin de production}

L'estimation des besoins pour la production et la croissance repose sur la quantité de $\mathrm{P}$ corporel, la quantité présente dans les produits de la conception et sécrétée via le lait.

\section{- Croissance corporelle}

La quantité de $\mathrm{P}$ corporel est décrite chez des animaux de 10 à $115 \mathrm{~kg}$ en fonction du poids à partir de données de la bibliographie. La dérivée première de ce polynôme permet de calculer la rétention de $\mathrm{P}$ par unité de gain de poids.

La même équation est utilisée pour estimer la rétention corporelle de $\mathrm{P}$ pour la truie en gestation. Faute de données expérimentales suffisantes, il est actuellement encore impossible de rendre compte de l'influence de la croissance différentielle des os et des tissus mous sur $\mathrm{P}$ corporel. Nous estimons ne pas disposer de données expérimentales suffisantes concernant la rétention (accrétion moins résorption) osseuse en relation avec le rang de gestation. De plus, nous ignorons presque totalement dans quelle mesure les réserves osseuses sont mobilisées pendant la lactation et l'impact de cette possible déminéralisation sur le besoin en $\mathrm{P}$ pendant la gestation suivante. Jongbloed et al (1999) différencient la quantité de P retenu dans les os et les tissus mous. Ils proposent une rétention de $\mathrm{P}$ dans les os maternels décroissant de 1,5 à $0,1 \mathrm{~g}$ par jour de la première à la cinquième gestation mais ne justifient pas l'origine de ces valeurs.

\section{- Produits de la conception}

La rétention de $\mathrm{P}$ dans les produits de la conception (placenta, fœetus) est proportionnelle à la rétention protéique, en considérant que les protéines contiennent $0,96 \%$ de P. La quantité de protéines dans les produits de la conception est estimée à partir d'équations de Gompertz établies par Noblet et al (1985). Contrairement à Jongbloed et al (1999), nous ne calculons pas spécifiquement la rétention de $\mathrm{P}$ dans l'utérus et la mamelle, considérant la croissance de ces organes comme partie intégrante du gain de poids de la truie.

\section{- Lactation}

Le besoin en $\mathrm{P}$ pour la lactation est calculé à partir de la production laitière, en considérant que la teneur en $\mathrm{P}$ du lait est de 1,55 g / kg (Guéguen et Perez 1981). La production de lait est fonction du nombre de porcelets allaités et de leur vitesse de croissance (Noblet et Etienne 1989). Cette approche diffère de celle proposée par Jongbloed et al (1999) qui estiment la quantité de $\mathrm{P}$ sécrétée via le lait à partir du besoin en $\mathrm{P}$ des porcelets en attribuant une valeur de 0,91 à la digestibilité de $\mathrm{P}$ du lait de truie. Ces auteurs estiment le contenu en $\mathrm{P}$ corporel du porcelet allaité à partir des 
Tableau 6. Estimation du besoin en $P$ digestible apparent du porcelet sevré et du porc en engraissement.

\begin{tabular}{|c|c|c|c|c|c|c|c|}
\hline Poids vif (kg) & 10 & 20 & 30 & 50 & 70 & 100 & 115 \\
\hline Vitesse de croissance $(\mathrm{kg} / \mathrm{j})$ & 0,40 & 0,50 & 0,60 & 0,75 & 0,85 & 0,90 & 0,85 \\
\hline Consommation d'aliment $(\mathrm{kg} / \mathrm{j})$ & 0,54 & 0,90 & 1,29 & 1,88 & 2,38 & 2,97 & 2,98 \\
\hline \multirow[t]{2}{*}{ Indice de consommation } & 1,35 & 1,80 & 2,15 & 2,50 & 2,80 & 3,30 & 3,50 \\
\hline & \multicolumn{7}{|c|}{$P$ digestible $(g / \mathrm{j})$} \\
\hline Entretien & 0,10 & 0,20 & 0,30 & 0,50 & 0,70 & 1,0 & 1,2 \\
\hline Croissance & 2,1 & 2,7 & 3,1 & 3,9 & 4,3 & 4,4 & 4,0 \\
\hline \multirow[t]{2}{*}{ Total } & 2,2 & 2,9 & 3,4 & 4,4 & 5,0 & 5,4 & 5,2 \\
\hline & \multicolumn{7}{|c|}{$P$ digestible ( $\mathrm{g} / \mathrm{kg}$ aliment) } \\
\hline Total & 4,2 & 3,2 & 2,7 & 2,3 & 2,1 & 1,8 & 1,7 \\
\hline
\end{tabular}

équations établies pour les porcs en croissance. De plus, ils considèrent que la truie en lactation mobilise $0,8 \mathrm{~g}$ de $\mathrm{P}$ par jour à partir de ses réserves osseuses. Dans notre approche cette déminéralisation osseuse n’est pas prise en compte, faute de références suffisantes dans la littérature.

\section{2 / Quelques exemples d'esti- mation du besoin en $P$ diges- tible apparent suivant le stade physiologique}

Des exemples de calcul du besoin en $P$ digestible apparent sont présentés aux tableaux 6, 7 et 8 pour respectivement sement, la truie en lactation et la truie en gestation. Il s'agit d'exemples calculés à partir de caractéristiques et performances des animaux spécifiées dans les tableaux. Pour des animaux présentant d'autres caractéristiques, les calculs le porcelet sevré et le porc en engrais-

doivent être adaptés au moyen des équations présentées au tableau 5.

\section{a) Porcelet sevré et porc en engrais-} sement

Le besoin en $\mathrm{P}$ digestible apparent augmente de 2,2 g / jour chez des porcelets sevrés pesant $10 \mathrm{~kg}$ et présentant une vitesse de croissance de $400 \mathrm{~g} /$ jour, à 5,4 g / jour chez des porcs pesant $100 \mathrm{~kg}$ avec une vitesse de croissance de 900 g / jour. Pour un indice de consommation augmentant de 1,35 chez le porcelet de $10 \mathrm{~kg}$ à 3,30 chez le porc en finition, le besoin en $\mathrm{P}$ digestible apparent diminue progressivement de 4,2 à 1,8 g $\mathrm{P}$ digestible / kg d'aliment lorsque le poids des animaux augmente de 10 à $100 \mathrm{~kg}$. Ces estimations sont très proches de celles calculées selon l'approche proposée par Jongbloed et al (1999) pour des porcs maigres.

Tableau 7. Estimation du besoin en P digestible apparent de la truie en lactation selon la taille de la portée et la quantité d'aliment consommée par la truie(1).

\begin{tabular}{|c|c|c|c|c|c|c|}
\hline \multirow{2}{*}{$\begin{array}{c}\text { Croissance de la } \\
\text { portée }(\mathrm{g} / \mathrm{j})\end{array}$} & 1750 & 2000 & 2250 & 2500 & 2750 & 3000 \\
\hline & \multicolumn{6}{|c|}{ P digestible $(\mathrm{g} / \mathrm{j})$} \\
\hline Entretien & 2,0 & 2,0 & 2,0 & 2,0 & 2,0 & 2,0 \\
\hline $\mathrm{P}$ sécrété via le lait & 9,5 & 10,8 & 12,2 & 13,5 & 14,9 & 16,2 \\
\hline \multirow[t]{2}{*}{ Total } & 11,5 & 12,8 & 14,2 & 15,5 & 16,9 & 18,2 \\
\hline & \multicolumn{6}{|c|}{$P$ digestible (g/ kg aliment) } \\
\hline $4 \mathrm{~kg}$ aliment / j & 2,9 & 3,2 & 3,5 & 3,9 & 4,2 & 4,6 \\
\hline $5 \mathrm{~kg}$ aliment $/ \mathrm{j}$ & 2,3 & 2,6 & 2,8 & 3,1 & 3,4 & 3,6 \\
\hline $6 \mathrm{~kg}$ aliment $/ \mathrm{j}$ & 1,9 & 2,1 & 2,4 & 2,6 & 2,8 & 3,0 \\
\hline \multirow[t]{2}{*}{$7 \mathrm{~kg}$ aliment $/ \mathrm{j}$} & 1,6 & 1,8 & 2,0 & 2,2 & 2,4 & 2,6 \\
\hline & \multicolumn{6}{|c|}{ Déminéralisation osseuse ( $\%$ de la réserve initiale) $)^{(2)}$} \\
\hline $4 \mathrm{~kg}$ aliment / j & & 3 & 8 & 12 & 17 & 22 \\
\hline $5 \mathrm{~kg}$ aliment $/ \mathrm{j}$ & & & & 2 & 7 & 11 \\
\hline $6 \mathrm{~kg}$ aliment / j & & & & & & 1 \\
\hline
\end{tabular}

(1) Pour une truie pesant $200 \mathrm{~kg}$.

(2) Pour un aliment contenant $3 \mathrm{~g} \mathrm{P}$ digestible apparent / kg distribué pendant une lactation de 28 jours, en considérant que le $\mathrm{P}$ corporel représente $0,5 \%$ du poids vif et que $80 \%$ du $\mathrm{P}$ corporel est contenu dans les os.

\section{b) Truie en lactation}

Dans l'exemple présenté au tableau 7, le besoin en $\mathrm{P}$ digestible apparent de la truie en lactation augmente de 11,5 à 18,2 g / jour lorsque la vitesse de croissance de la portée augmente de 1750 à 3000 g / jour. Si l'aliment est trop peu concentré en $\mathrm{P}$ digestible apparent, une perte d'appétit peut être à l’origine d'un bilan négatif. Le phosphore secrété via le lait peut alors être mobilisé à partir des réserves osseuses. Dans l'exemple présenté au tableau 7, une truie consommant quotidiennement $5 \mathrm{~kg}$ d'un aliment contenant $3 \mathrm{~g}$ de $\mathrm{P}$ digestible apparent / $\mathrm{kg}$ présente un bilan de $P$ négatif dès que la vitesse de croissance de la portée atteint 2400 g par jour, soit une portée d'environ 9-10 porcelets. En considérant que $\mathrm{P}$ corporel, localisé à $80 \%$ dans les os, représente $0,5 \%$ du poids vif, on estime que, pour une durée de lactation de 28 jours, jusqu’à $22 \%$ de la réserve osseuse initiale pourraient être mobilisés dans le cas extrême de l'ingestion quotidienne de $4 \mathrm{~kg}$ d'un aliment contenant 3 g de $\mathrm{P}$ digestible / kg, jusqu'à une truie allaitant une portée de 12 porcelets présentant une vitesse de croissance de 250 g/jour (tableau 7). Selon Jongbloed et al (1999), la mobilisation des réserves osseuses atteindrait 22,8 g de $P$ pour une lactation de 28 jours, soit près de $3 \%$ de la réserve osseuse initiale d'une truie de $200 \mathrm{~kg}$. Pour un aliment contenant $3 \mathrm{~g}$ de $\mathrm{P}$ digestible / kg, cette estimation correspond à une truie présentant un appétit moyen et allaitant une portée de taille moyenne (tableau 7).

\section{c) Truie en gestation}

Pendant les deux premiers tiers de la gestation, le besoin en $\mathrm{P}$ correspond en grande partie au besoin maternel d'entretien et de croissance (tableau 8). Au cours du dernier tiers de gestation, le besoin augmente en raison de la croissance des produits de la conception. En 
Tableau 8. Estimation du besoin en P digestible apparent de la truie en gestation selon le nombre de porcelets nés(1).

\begin{tabular}{|c|c|c|c|c|c|c|c|c|}
\hline \multicolumn{2}{|c|}{ Jour de gestation } & 0 & 28 & 56 & 84 & 100 & 105 & 110 \\
\hline & & \multicolumn{7}{|c|}{$P$ digestible $(g / j)$} \\
\hline \multirow{5}{*}{10 porcelets } & Entretien & 1,7 & 1,9 & 2,0 & 2,1 & 2,2 & 2,2 & 2,2 \\
\hline & Croissance maternelle & 2,1 & 2,1 & 2,0 & 2,0 & 2,0 & 2,0 & 2,0 \\
\hline & Foetus & 0,00 & 0,00 & 0,2 & 1,4 & 2,1 & 2,3 & 2,4 \\
\hline & Placenta & 0,00 & 0,01 & 0,05 & 0,03 & 0,03 & 0,02 & 0,02 \\
\hline & Total & 3,8 & 3,9 & 4,3 & 5,6 & 6,3 & 6,5 & 6,7 \\
\hline \multirow[t]{2}{*}{14 porcelets } & Foetus & 0,00 & 0,01 & 0,3 & 1,9 & 3,0 & 3,2 & 3,4 \\
\hline & Placenta & 0,00 & 0,01 & 0,06 & 0,04 & 0,03 & 0,03 & 0,03 \\
\hline \multirow{4}{*}{18 porcelets } & Total & 3,8 & 4,0 & 4,4 & 6,1 & 7,2 & 7,4 & 7,6 \\
\hline & Foetus & 0,00 & 0,01 & 0,4 & 2,5 & 3,8 & 4,1 & 4,3 \\
\hline & Placenta & 0,00 & 0,01 & 0,08 & 0,05 & 0,04 & 0,04 & 0,04 \\
\hline & Total & 3,8 & 4,0 & 4,5 & 6,7 & 8,0 & 8,4 & 8,6 \\
\hline & & \multicolumn{7}{|c|}{$P$ digestible ( $\mathrm{g} / \mathrm{kg}$ aliment) } \\
\hline 10 porcelets & Total & 1,3 & 1,4 & 1,5 & 1,9 & 2,2 & 2,3 & 2,3 \\
\hline 14 porcelets & Total & 1,3 & 1,4 & 1,6 & 2,1 & 2,5 & 2,6 & 2,7 \\
\hline 18 porcelets & Total & 1,3 & 1,4 & 1,6 & 2,3 & 2,8 & 2,9 & 3,0 \\
\hline
\end{tabular}

(1) Pour une truie en deuxième gestation pesant $172 \mathrm{~kg}$ en début de gestation, présentant une vitesse de croissance de 0,500 kg par jour et consommant $2,85 \mathrm{~kg}$ d'aliment par jour - les porcelets pèsent $1,4 \mathrm{~kg}$ à la naissance.

fin de gestation, le besoin dépend de la prolificité de la truie : il augmente de 6,7 à 8,6 g / jour pour une truie en deuxième gestation produisant une portée de respectivement 10 et 18 porcelets pesant $1,4 \mathrm{~kg}$ à la naissance. L'allure de cette courbe de besoin montre bien la possibilité de réduction des apports alimentaires de $\mathrm{P}$ en début de gestation. Toutefois, l'abaissement des apports dans ce cadre doit être mené avec prudence en raison de la possible surestimation de l'apport alimentaire de $\mathrm{P}$ digestible aux animaux à ce stade physiologique et du besoin, non comptabilisé, de restauration des réserves éventuellement mobilisées lors de la lactation précédente. Dans le cas extrême d'une déminéralisation représentant $22 \%$ des réserves osseuses pendant la gestation (tableau 7), 175 g de P digestible apparent devraient être apportés en plus à la truie, soit un apport quotidien supplémentaire de $2,3 \mathrm{~g}$ de $\mathrm{P}$ digestible apparent, si ces réserves doivent être restaurées en 75 jours. Dans l'exemple donné au tableau 8, la teneur en $\mathrm{P}$ digestible apparent de l'aliment distribué en début de gestation devrait alors être augmentée de 0,8 g / kg, soit une teneur qui reste inférieure à la teneur requise en fin de gestation.

\section{Conclusion}

Ce nouveau système d'évaluation de la valeur $\mathrm{P}$ des matières premières et des aliments et du besoin des animaux fondé sur la digestibilité apparente représente sans conteste un progrès par rapport au système antérieur qui reposait sur le phosphore total. Il permet de mieux ajuster les apports aux besoins des animaux et de mettre en œuvre des voies d'amélioration de la digestibilité de $\mathrm{P}$, notamment l'usage de phytase, et donc de réduire les apports alimentaires de $\mathrm{P}$ sans compromettre les performances des animaux. Cependant, des progrès sont encore possibles concernant l'estimation de la valeur $P$ des matières premières et des aliments, et l'estimation du besoin des animaux. L'impact de certains éléments constitutifs de la ration, comme les cations divalents et celui du stade physiologique des animaux sur la valeur $P$ des aliments et sur l'efficacité des phytases qui y sont introduites, devra être pris en compte. De plus, l'opportunité d'une correction de la digestibilité apparente par des pertes endogènes basales reste à évaluer. Ces questions peuvent être investiguées au moyen de l'analyse des nombreuses données publiées. Pour l'estimation du besoin en P de la truie en reproduction, un certain nombre d'approximations sont encore faites en raison de notre méconnaissance partielle des possibilités de mobilisation et de restauration de la réserve osseuse au cours du cycle de reproduction. Avant d'abaisser les apports excessifs de $\mathrm{P}$ à ces animaux, il sera nécessaire d'acquérir de nouvelles données expérimentales.

\section{Remerciements}

Les auteurs remercient Fabien Skiba d’Arvalis - Institut du végétal, pour leur avoir gracieusement confié sa base de données concernant la digestibilité de $\mathrm{P}$ des matières premières. Ils remercient également Léon Guéguen et Alain Pointillart pour la relecture de cet article.

\section{Références}

ARC, Agricultural Research Council, 1981. The nutrient requirements of pigs. CAB. Slough UK, 297p.

Augspurger N., Webel D., Lei X., Baker D., 2003. Efficacy of an E. coli phytase expressed in yeast for releasing phytate-bound phosphorus in young chicks and pigs. J. Anim. Sci., 81, 474-483.

Augspurger N.R., Spencer J.D., Webel D.M., Baker D.H., 2004. Pharmacological zinc levels reduce the phosphorus-releasing efficacy of phy- tase in young pigs and chickens. J. Anim. Sci., 82, 1732-1739.

Carlson D., Poulsen H.D., 2003. Phytate degradation in soaked and fermented liquid feed - effect of diet, time of soaking, heat treat- 
ment, phytase activity, $\mathrm{pH}$ and temperature. Anim. Feed Sci. Technol., 103,141-154.

CORPEN, 2003. Estimation des rejets d'azote, phosphore, potassium, cuivre et zinc des porcs. Corpen (Eds), Paris, France, 41p.

CVB, 2000. Veevoedertabel. Centraal Veevoederbureau, Lelystad, The Netherlands.

Düngelhoef M., Rodehutscord M., 1995. Effects of phytases on the digestibility of phosphorus in pigs. Übersicht. Tierernähr., 23, 133157.

Eeckhout W., De Paepe M., 1992. Phytase de blé, phytase microbienne et digestibilité apparente du phosphore d'un aliment simple pour porcelets. Rev. Agric., 45, 195-207.

Eeckhout W., De Paepe M., 1994. Total phosphorus, phytate-phosphorus and phytase activity in plant feedstuffs. Anim. Feed Sci. Technol., 47, 19-29.

Eeckhout W., De Paepe M., Warnants N., Bakaert H., 1995. An estimation of the minimal $\mathrm{P}$ requirements for growing-finishing pigs, as influenced by the Ca level of the diet. Anim. Feed Sci. Technol., 52, 29-40.

Engelen A.J., van der Heeft F.C., Randsdorp P. H.G., Smit E.L.C., 1994. Simple and rapid determination of phytase activity. J. AOAC Intern., 77, 760-764.

Fan M.Z., Archbold T., Sauer W.C., Lackeyram D., Rideout T., Gao Y., De Lange C. F.M., Hacker R.R., 2001. A novel methodology allows simultaneous mesurement of true phosphorus digestibility and the gastrointestinal endogenous phosphorus outputs in studies with pigs. J. Nutr., 131, 2388-2396.

Fan M.Z., Sauer W.C., 2002. Additivity of apparent ileal and fecal phosphorus digestibility values measured in single feed ingredients for growing-finishing pigs. Can. J. Anim. Sci., 82, 183-191.

Golovan S.P., Meidinger R.G., Ajakaiye A., Cottrill M., Wiederkehr M.Z., Barney D.J., Plante C., Pollard J.W., Fan M.Z., Hayes M.A., Laursen J., Hjorth J.P., Hacker R R., Phillips J. R., Forsberg C.W., 2001. Pigs expressing salivary phytase produce low-phosphorus manure. Nature Biotech., 19, 741-745.

Guéguen L., Perez J.M., 1981. A re-evaluation of recommended dietary allowances of calcium and phosphorus for pigs. Proc. Nutr. Soc., 40, 273-278.

Guéguen L., Pointillart A., 1986. Alimentation minérale. In: J.M. Perez, P. Mornet, A. Rérat (eds), Le porc et son élevage, bases scientifiques et techniques, INRA, Maloine (Eds), Paris, France, 297-322.

INRA, 1989. L'alimentation des animaux monogastriques : porc, lapin, volailles. Seconde édition révisée, INRA, Paris, France, 282p.

INRA-AFZ, 2004. Tables de composition et de valeur nutritive des matières premières destinées aux animaux d'élevage : porcs, volailles, bovins, ovins, caprins, lapins, chevaux, poissons. D. Sauvant, J.-M. Perez, G. Tran (eds), $2^{\mathrm{e}}$ édition révisée, INRA, Paris, 301p.

Jean dit Bailleul P., Bernier J.F., Van Milgen J., Sauvant D., Pomar C., 2001. Méta-analyse de l'effet de la phytase dans les aliments pour porcs. Journ. Rech. Porcine. Fr., 33, 43-48.
Johansen K., Poulsen H.D., 2003. Substitution of inorganic phosphorus in pig diets by microbial phytase supplementation - a review. Pig News Inform., 24, 77N-88N

Jongbloed A.W., Everts H., Kemme P.A., Mroz, Z., 1999. Quantification of absorbability and requirements of macroelements. In: I. Kyriazakis (eds), Quantitative biology of the pig, CAB International, Wallingford, UK, 275298.

Jongbloed A.W., Mroz Z., van der WeijJongbloed R., Kemme P. A., 2000. The effects of microbial phytase, organic acids and their interaction in diets for growing pigs. Livest. Prod. Sci., 67, 113-122.

Jongbloed A.W., van Diepen J.T.M., Kemme P.A., Mroz J., 2004. Efficacy of microbial phytase on mineral digestibility in diets for gestating and lactating sows. Livest. Prod. Sci., 91, 143155.

Kemme P.A., Jongbloed A.W., Mroz Z., Beynen A.C., 1997a. The efficacy of Aspergillus niger phytase in rendering phytate phosphorus available for absorption in pigs is influenced by pig physiological status. J. Anim. Sci., 75, 21292138.

Kemme P.A., Radcliffe J.S., Jongbloed A.W. Mroz Z., 1997b. Factors affecting phosphorus and calcium digestibility in diets for growingfinishing pigs. J. Anim. Sci., 75, 2139-2146.

Kemme P.A., Jongbloed A.W., Mroz Z., Kogut J., Beynen A.C., 1999. Digestibility of nutrients in growing-finishing pigs is affected by Aspergillus niger phytase, phytate and lactic acid levels: 1. Apparent ileal digestibility of amino acids. Livest. Prod. Sci., 58, 107-117.

Kornegay E.T., 2001. Digestion of phosphorus and other nutrients: the role of phytases and factors influencing their activity. In: M.R. Bedford and G.G. Partridge (eds), Enzymes in Farm Animal Nutrition, CAB International, Wallingford, UK, 237-271.

Larsen T., Skoglund E., Sandberg A.S., Engberg R.M., 1999. Soaking and pelleting of pig diets alters the apparent absorption and retention of minerals. Can. J. Anim. Sci., 79, 477-483.

Liu J., Bollinger D.W., Ledoux D.R., Ellersieck M.R., Veum T.L., 1997. Soaking increases the efficacy of supplemental microbial phytase in a low-phosphorus corn-soybean meal diet for growing pigs. J. Anim. Sci., 75, 12921298.

Näsi J.M., Helander E.M., Partanen K.H., 1995. Availability for growing pigs of minerals and protein of a high phytate-barley-rapeseed meal diet treated with Aspergillus niger phytase or soaked with whey. Anim. Feed Sci. Technol., $56,83-98$.

Noblet J., Close W.H., Heavens R.P., 1985. Studies on the energy metabolism of the pregnant sow. 1. Uterus and mammary tissue development. Br. J. Nutr., 53, 251-265.

Noblet, J., Etienne M., 1989. Estimation of sow milk nutrient output. J. Anim. Sci., 67, 3352-3359.

NRC, 1998. Nutrient requirements of swine. Tenth revised edition. National Academy Press, Wahsington DC, 211p.

Nys Y., Frapin D., Pointillart A., 1996. Occurrence of phytase in plants, animals and microorganisms. In: M.B. Coelho, E.T. Korne- gay (eds), Phytase in Animal Nutrition and Waste Management, BASF corporation, 213-236.

Omogbenigun F.O., Nyachoti C.M. Slominski B.A., 2003. The effect of supplementing microbial phytase and organic acids to corn-soybean based diet fed to early-weaned pigs. J. Anim. Sci., 81, 1806-1813.

Pointillart A., 1991. Enhancement of phosphorus utilization in growing pigs fed phytate-rich diets by using rye bran. J. Anim. Sci., 69, 11091115.

Pointillart A., 1994. Phytates, phytases : leur importance dans l'alimentation des monogastriques. INRA Prod. Anim., 7, 29-39.

Poulsen H.D., Jongbloed A.W., Latimier P., Fernandez J.A., 1999. Phosphorus consumption, utilisation and losses in pig production in France, The Netherlands and Denmark. Livest. Prod. Sci., 58, 251-259.

Qian H., Kornegay E.T., Conner D.E.J., 1996. Adverse effects of wide calcium: phosphorus ratios on supplemental phytase efficacy for weanling pigs fed two dietary phosphorus levels. J. Anim. Sci., 74, 1288-1297.

Radcliffe J.S., Zhang Z.B., Kornegay E.T., 1998. The effects of microbial phytase, citric acid and their interaction in a corn-soybean meal-based diet for weanling pigs. J. Anim. Sci., 76, 1880-1886.

Rapp C., Lantzsch H.J., Drochner W., 2001. Hydrolysis of phytic acid by intrinsic plant or supplemented microbial phytase (Aspergillus niger) in the stomach and small intestine of minipigs fitted with re-entrant cannulas. 2 Phytase activity. J. Anim. Physiol. Anim. Nutr. 85, 414-419.

Rodehutscord M., Krause G., Pfeffer E., 1999. Effect of body weight on phosphorus digestibility and efficacy of a microbial phytase in young pigs. Arch. Anim. Nutr., 52, 139-153.

Sauveur B., Perez J.M., 1989. Alimentation minérale des animaux monogastriques. In L'alimentation des animaux monogastriques porc, lapin, volailles. $2^{\mathrm{e}}$ ed., INRA Editions, Paris, France, 23-31.

Schlemmer U., Jany K. D., Berk A., Schulz E. Rechkemmer G., 2001. Degradation of phytate in the gut of pigs - pathway of gastrointestinal inositol phosphate hydrolysis and enzymes involved. Arch. Anim. Nutr., 55, 255 280

Sève B., Tran G., Jondreville C., Skiba F., van Cauwenberghe S., Bodin J.-C., Langer S., 2000. Measuring ileal basal endogenous losses and digestive utilisation of amino acids through ileorectal anastomosis in pigs: ring test between three laboratories. In: Proc. 8th Intern. Symp. on Digestive Physiology in Pigs, 20-22 June 2000, Uppsala, Sweden.

Shen Y., Fan M., Ajakaiye A., Archbold T., 2002. Use of the regression analysis technique to determine the true phosphorus digestibility and the endogenous phosphorus output associated with corn in growing pigs. J. Nutr., 132, 11991206.

Skiba F., Callu P., Castaing J., Paboeuf F. Chauvel J., Jondreville C., 2004. Variabilité intra matière première de la digestibilité du phosphore des céréales et du pois chez le porc en croissance. Journ. Rech. Porcine Fr., 36, 9-16.

Skoglund E., Larsen T., Sandberg A. S., 1997. Comparison between steeping and pelleting a 
mixed diet at different calcium levels on phytate degradation in pigs. Can. J. Anim. Sci., 77, 471477.

Spencer J. D., Allee G. L., Sauber T. E., 2000. Phosphorus availability and digestibility of normal and genetically modified low-phytate corn for pigs. J. Anim. Sci., 78, 675-681.

Veum T.L., Ledoux D.R., Raboy V., Ertl D. S., 2001. Low-phytic acid corn improves nutrient utilization for growing pigs. J. Anim. Sci., 79, 2873-2880.
Veum T.L., Ledoux D.R., Bollinger D.W., Raboy V., Cook A., 2002. Low-phytic acid barley improves calcium and phosphorus utilization and growth performance in growing pigs. J. Anim. Sci., 80, 2663-2670.

Weremko D., Fandrejewski H., Zebrowska T., Han K., Kim J.H., Cho W.T., 1997. Bioavailability of phosphorus in feeds of plant origin for pigs. A review. Asian-Austr. J. Anim. Sci., 10, 551-566.

Zimmermann B., Lantzsch H.J., Mosenthin R., Biesalski H.K., Drochner W., 2002.
Comparative evaluation of the efficacy of cereal and microbial phytases in growing pigs fed diets with marginal phosphorus supply. J. Sci. Food Agric., 82, 1298-1304.

Zimmermann B., Lantzsch H.J., Mosenthin R., Biesalski H.K., Drochner W., 2003. Additivity of the effect of cereal and microbial phytases on apparent phosphorus absorption in growing pigs fed diets with marginal P supply. Anim. Feed Sci. Technol., 104, 143-152.

\section{Résumé}

Limiter les apports alimentaires de phosphore $(P)$ aux porcs pour en réduire les rejets suppose la mise en ouvre de stratégies d'alimentation permettant de mieux ajuster les apports aux besoins des animaux et mettant en ouvre des voies d'amélioration de la disponibilité de $P$. Un système d'évaluation de la valeur $P$ des aliments et d'estimation des besoins des animaux s'appuyant sur la digestibilité fécale apparente est proposé. L'impact de facteurs de variation de la digestibilité de $P$ tels que la proportion de $P$ phytique dans $P$ total et la présence de phytases, végétales ou microbiennes, est quantifié. Le nouveau système représente un progrès par rapport à la formulation sur la base de $\mathbf{P}$ total, même si l'impact d'autres facteurs de variation identifiés reste à quantifier. Les bases de l'estimation factorielle du besoin en $P$ sont exprimées conformément au concept de digestibilité apparente. Des exemples d'estimation du besoin en $P$ digestible apparent de porcelets, porcs en engraissement et truies en lactation ou en gestation selon leurs caractéristiques et performances sont présentés. Des marges de progrès sont encore possibles pour réduire les apports alimentaires de $P$ aux truies, mais elles devront s'appuyer sur l'acquisition de nouvelles données expérimentales concernant les possibilités de mobilisation et de restauration de la réserve osseuse au cours du cycle de reproduction.

\section{Abstract}

\section{Phosphorus in pig nutrition}

In order to reduce $\mathbf{P}$ excreted by pigs, $\mathbf{P}$ dietary supply should be better adjusted to their requirements and strategies to improve $\mathbf{P}$ availability should be implemented. A feeding system relying on $P$ apparent digestibility is proposed. The impact of the proportion of phytic $\mathbf{P}$ in total $\mathbf{P}$ and of plant and microbial phytase on $\mathbf{P}$ digestibility was quantified. Apparent digestibility was a more efficient approach than total $P$ to formulate pig diets, even if the impact of other known factors of variation of $P$ digestibility remains to be quantified. The bases for the factorial determination of apparent digestible $P$ requirements by weaned piglets, fattening pigs and lactating and gestating sows according to their characteristics and performance were updated. A reduction of $\mathbf{P}$ supply to sows is still possible, but new experimental data regarding the management of bone stores throughout lactation and gestation are required.

JONDREVILLE C., DOURMAD J.-Y., 2005. Le phosphore dans la nutrition des porcs. INRA Prod. Anim., 18, $183-192$. 